Apidologie, 1973, 4 (1), 41-44.

\title{
DROHNEN VON APIS CERANA FABR. AUF EINEM DROHNENSAMMELPLATZ
}

Mâles d'Apis cerana Fabr. sur un lieu de rassemblement de mâles

Friedrich RUTTNER

Institut für Bienenkunde, Oberursel

SUMMARY

DRONES OF APIS CERANA AT A CONGREGATION AREA

In one case, drones of Apis cerana indica were caught at a congregation area near Frankfurt together with mellifera drones. Their frequency at the congregation area was about the same as their estima' ed frequency in the drone population of the area.

\section{ZUSAMMENFASSUNG}

Auf einem Sammelplatz bei Frankfurt/M stammten $53(=2,12 \%)$ von 2.599 gefangenen Drohnen aus 2 in der Nähe aufgestellten Völkern von Apis cerana. Da die Zahl der Bienenvölker (Apis mellifica) in der Umgebung etwas über 100 betrug, entsprach dieser Anteil ziemlich genau der Häufigkeit der Cerana-Drohnen in der Drohnenpopulation des Gebietes.

Drohnen ( $(\widehat{\delta})$ der Westlichen Honigbiene (Apis mellifica L.) versammeln sich auf ihren Hochzeits flügen über bestimmten Stellen im Gelände (“ Drohnensammelplätze »). Die Entfernung zwischen dem Standort der $\widehat{\partial} \widehat{0}$ und den Sammelplätzen beträgt in der Regel 0-3 km, ein geringer Prozentsatz der $\widehat{o} \sigma^{*}$ eines Sammelplatzes kann aber von Bienenständen in mehr als $6 \mathrm{~km}$ 
Entfernung stammen. Die Flugrichtung zu den Sammelplätzen wird offensichtlich auf Grund eines spezifischen Orientierungssystems gewählt; welche Faktoren die Tiere veranlassen, über diesen deutlich begrenzten PIätzen zu verweilen, ist derzeit noch nicht bekannt.

Bisher konnten $\not \partial \alpha$ von sämtlichen europäischen und auch von afrikanischen Rassen (A. mellifica carnica, ligustica, mellifica, intermissa), sowie von selektierten Kunstrassen und von Mutanten (aurea, cordovan) auf Sammelplätzen festgestellt werden. Das Aufsuchen von Sammelplätzen scheint also ein regulärer Abschnitt im Paarungsverhalten der $\hat{o} \partial \hat{\alpha}$ von $A$. mellifica zu sein (H. und F. RutTnER, 1972).

Es ist darum von Interesse, das Verhalten anderer Apis-Arten beim Hochzeits flug zu untersuchen. Für Apis cerana konnten kürzlich die ersten Beobachtungen über die Hochzeitsflüge der Königinnen und über die Spermaübertragung durchgeführt werden (F. RutrNer, J. Woyke und N. KoeniGER, 1972). Im Sommer 1972 ist es gelungen, auch über das Flugverhalten der Drohnen Erfahrungen zu sammeln.

\section{METHODIK}

Am 15. Juni wurden in $300 \mathrm{~m}$ Entfernung von einem stark frequentierten Drohnensammelplatz am Rande des Städtchens Kronberg/Taunus 2 Völker von Apis cerana indica aufgestellt. Der Sammelplatz befindet sich über der Sohle eines kleinen, in N-S-Richtung ziehenden Tälchens, über einer sumpfigen Wiese. An den beidseitigen Hängen des Tälchens Gärten mit einigen, z.T. höheren Bäumen. Der Standort der cerana $\hat{o}_{\hat{o}} \hat{o}$ lag südlich (talauswärts) vom Sammelplatz, am Westhang des Tälchens. Zwischen Standort und Sammelplatz befindet sich ein Bestand alter, hoher Edelkastanien. Die Begrenzung des Sammelplatzes konnte in mehreren Versuchen eindeutig markiert werden : $100 \mathrm{~m}$ (in der Längsachse des Tälchens) $\times 50 \mathrm{~m}$.

Die beiden Völker von $A$. cerana waren im Frühjahr 1971 mit freundlicher Unterstützung von Dr. M. Rashid Khan durch Dr. N. Koeniger von der Beekeeping Section der Tarnab Farm, Peshawar (Pakistan) nach Oberursel gebracht worden. Die Uberwinterung erfolgte im Flugraum des Institutes. Zur Zeit des Versuches belagerten beide Völker 6 Waben, sie besassen aber etwa ebensoviele ${ }^{\star} \precsim \alpha$ wie ein normal starkes Mellifica-Volk um diese Zeit (je etwa 1.000). Der Fang der $\delta^{+}{ }^{+}$erfolgte in der üblichen Weise mittels Netzen, nach Köderung durch eine an einem Ballon befestigte Königin. Da beide Arten denselben Sexuallockstoff besitzen (BUTLER, Calam und Callow, 1967) und Cerana-Königinnen für Mellifica- $0^{*}{ }^{*}$ attraktiv sind - wenn auch weniger stark wie für $\delta 3$ der eigenen Art (RutTNer und Kaissling, 1968) - erschien dieses Verfahren vertretbar, obwohl das Verhalten der Cerana-ș ginnen bisher noch nicht quantitativ untersucht worden ist.

Die Identifizierung der Cerana- $\widehat{\delta}^{\circ}{ }^{\dagger}$ gelingt schon mit blossen Auge, wenn auch an getöteten

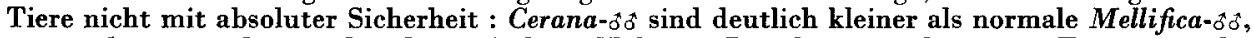
ganz schwarz und von charakteristischem Habitus. Da aber an sehr guten Fangtagen die Identifizierung an Ort und Stelle nicht möglich ist (einmal wurden im Verlaufe von 2 Stunden etwa 2.000 के ihrer Grösse sortiert. Aus der Gruppe der kleinen ${ }^{*}{ }^{*}$ wurden kleine, in Arbeiterzellen aufgezogene Mellifica- $\hat{o}^{*}$ mittels des Geäders am Hinterflügel bestimmt und ausgeschieden. Die verlängerte Radialis-Ader am Hinter flügel ist ein absolut verlässliches Merkmal für Cerana- đơ ơ (Abb. 1).

\section{ERGEBNISSE}

An 4 Fangtagen wurden insgesamt 2599 ภิวิ gefangen.

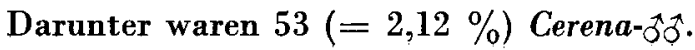



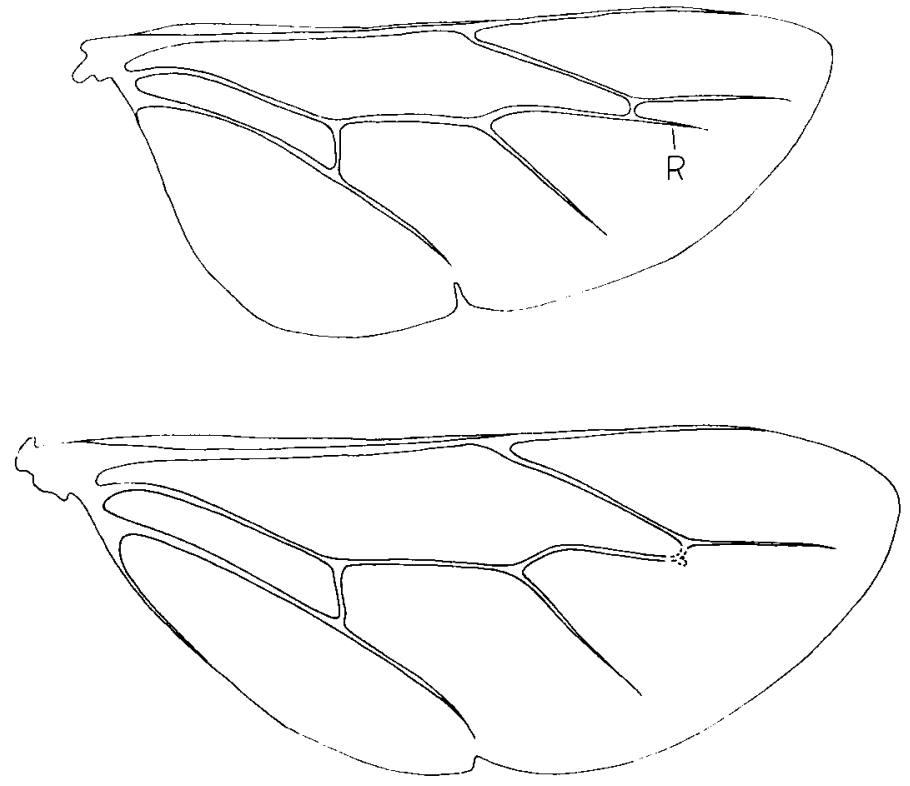

ABB. 1. - Oben : Hinterflügel von A. cerana mit verlängerter Radialis ( $R)$. Unten : Hinterflügel von A. mellifica.

Die Ansatzstelle der Radialis -Verlängerung ist variabel (rund, eckig oder Stummel).

FIG. 1. - En haut : aile postérieure d'Apis cerana avec artère radiale prolongée (R). En bas : aile postérieure d'Apis mellifica.

Le point d'attache du prolongement de l'artère radiale est variable (rond, anguleux, tronqué).

In unmittelbarer Nähe des Sammelplatzes (dh. in einer Entfernung von etwa $300 \mathrm{~m}$ ) befanden sich zu dieser Zeit zwei grössere Bienenstände mit je etwa 50 Bienenvölkern, und in der weiteren Umgebung vermutlich einige Stände mit wenigen Völkern, zusammen also mehr als 100 Bienenvölker. Die 2 Cerana-Völker hatten somit einen Anteil von etwas weniger als $2 \%$ an dieser Population. Wie oben erwähnt, entsprach ihr Drohnenbestand etwa dem eines normalen Volkes.

Mit 2,12\% waren also die Cerana- ở am Sammelplatz entsprechend ihrer Häufigkeit in diesem Areal repräsentiert. Aus dieser Beobachtung kann geschlossen werden, dass sich die $\delta \hat{\sigma}$ von Apis cerana indica bei ihren Aus flügen ähnlich verhalten wie die $\delta \widehat{\partial}$ von $A$ pis mellifica und dass sie sich offenbar nach denselben Reizparametern orientieren wie diese. 


\section{DANKSAGUNG}

Dr. N. Koeniger und J. Kefuss danke ich bestens für ihre Mitarbeit.

Die Beschaffung und Haltung der Cerana-Völker wurde durch eine Beihilfe der Deutschen Forschungsgemeinschaft ermöglicht.

\section{RESUME}

On sait que les mâles d'Apis mellifica (abeille occidentale) se rassemblent en des endroits bien déterminés, éloignés parfois de plusieurs $\mathrm{km}$ de leur ruche d'origine, au moment des vols de fécondation. Depuis que ce phénomène est connu il a été possible de montrer que ce type de comportement se retrouve dans toutes les races géographiques européennes et africaines, y compris chez des mutants tels que cordovan. Il était donc intéressant de chercher à savoir si, sur ce point particulier, le comportement des mâles d'une autre espèce, en l'occurence Apis cerana Fabr. (Abeille orientale) est le même que celui d'Apis mellifica. Dans ce but, au mois de juin 1972, à $300 \mathrm{~m}$ environ d'un lieu de rassemblement de mâles très fréquenté, situé en bordure de la petite ville de Kronberg/Taunus, on a mis en place deux colonies d'Apis cerana provenant du Pakistan. Ces deux colonies étaient fortes de 6 cadres et avaient élevé un nombre de mâles aussi important qu'une forte colonie d'Apis mellifica.

A l'endroit où se rassemblent les mâles on a effectué des captures de ceux-ci par la méthode des leurres (reine encagée) suspendus à un ballon. Les mâles pris dans les filets ont été examinés du point de vue des caractères morphologiques. Une différence très caractéristique entre les mâles des deux espèces (nervation de l'aile postérieure) a permis de faire le tri d'une façon très sûre (Fig. 1).

Par cette méthode on a découvert que sur 2.599 mâles capturés 53 appartenaient à l'espèce Cerana, soit 2,12\%. Étant donné que dans les environs du lieu de rassemblement de mâles il se trouvait au moment des expériences environ 100 ruches, les captures effectuées sont dans un rapport numérique qui prouve que les mâles des deux espèces se comportent, sur ce point, de façon analogue.

\section{LITERATUR}

Butler C.G., Calam D.H., Callow R.K., 1967. Attraction of Apis mellifera drones by the odours of the queens of two other species of honeybees. Nature, London 213,423-424.

Ruttner F., Kaissling K.-E., 1968. Über die interspezifische Wirkung des Sexuallockstoffes von Apis mellifica und Apis cerana. Z. vergl. Physiol. 59, 362-370.

Ruttner F., Woyke J., Koeniger N., 1972. Reproduction in Apis cerana. J. Apicult. Res. 11, $141-146 ; 12(1)$.

RuttNer H., RutTNer F., 1972. Untersuchungen über die Flugaktivität und das Paarungsverhalten der Drohnen. V. Drohnensammelplätze und Paarungsdistanz. Apidologie 3, 203-233. 